\title{
Position, direction, and their perceptual integrality
}

\author{
HERBERT H. CLARK and HIRAM H. BROWNELL \\ Stanford University, Stanford, Califormia 94305
}

\begin{abstract}
Subjects were shown an arrow inside a rectangle and were asked to decide on either its vertical direction (up vs. down) or its height (high vs. low) as quickly as possible. Whenever vertical direction was criterial, height was irrelevant, and vice versa. In four conditions, the irrelevant dimension (1) did not vary, (2) covaried positively with the criterial dimension, up with high and down with low, (3) covaried negatively with the criterial dimension, up with low and down with high, or (4) varied orthogonally with the criterial dimension. Height and vertical direction satisfied one of $W$. R. Garner's prerequisites for "integral" dimensions in that Condition 4 took longer than Condition 1. But Condition 2 was faster than 1, and 3 was slower than 1 , a pattern unlike those of other known integral dimensions. The positive correlation in Condition 2 facilitates, and the negative correlation in 3 interferes, it is argued, because height and vertical direction have interpretations with components in common or in conflict.
\end{abstract}

Garner and Felfoldy (Felfoldy \& Garner, 1971; Garner, 1972, 1974; Garner \& Felfoldy, 1970) have argued that two attributes of a single stimulus can be "integral," "separable," or somewhere in between. The lightness and saturation of a single color chip, for example. are integral. When subjects were asked to sort color chips on the basis of saturation, they couldn't ignore irrelevant variation in lightness, or vice versa (Garner \& Felfoldy, 1970). This is a defining characteristic of integral dimensions. Garner (1970) has gone on to propose that one attribute will be integral with another "if in order for one dimension to exist the other must be specified" (p. 146). For example, it is impossible to specify the lightness of a color chip without specifying its saturation, and vice versa. and so lightness and saturation should be symmetrically integral. Indeed, subjects found it as hard to ignore one attribute as the other in Garner and Felfoldy's experiment.

As Garner (1974) has noted, however, integrality can be asymmetrical when one of two dimensions requires the specification of the other, but not vice versa. For illustration, Garner pointed to an experiment by Wood and Day (cited in Garner, 1974) in which subjects had to discriminate among the two syllables / ba/ and /ga/ spoken at either a high or a low pitch. Since every syllable must have pitch, pitch ought to be integral with syllabicity, but since not every audible stimulus with pitch is a syllable, syllabicity ought not to be integral with pitch. Consistent with this analysis, Wood and Day's subjects in judging pitch were able to ignore irrelevant variation in syllabicity, but in judging syllables weren't able to ignore irrelevant variation in pitch.

This research was supported in part by Grant MH 20021 from the National Institute of Mental Health. We thank William P. Banks, Wendell R. Garner, James R. Pomerantz, and Edward E. Smith for their suggestions at various stages of this research. Hiram H. Brownell is now at The Johns Hopkins University.
Integrality has been found to take other forms as well (see Pomerantz \& Garner, 1973; Pomerantz \& Sager. 1975).

In this study, we consider a form of integrality unlike others Garner has catalogued so far, a type we turned up in a study on judgments of height and vertical direction (Clark \& Brownell, 1975). In that study, we confronted subjects with a single arrow inside of a rectangle. The arrow pointed either up or down, and it was either high or low in the rectangle. The four configurations used are shown in Figure 1. When subjects had to decide whether the arrow pointed up or down, they were faster for the UP arrow when it was high in the rectangle (configuration a) than when it was low (b). In contrast, they were faster on the DOWN arrow when it was low (d) than when it was high (c). The subjects were faster when the direction of the arrow (up vs. down) was in

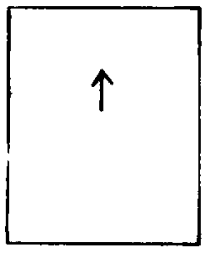

a

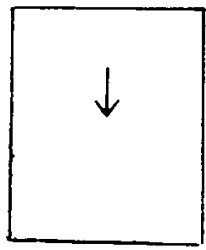

C
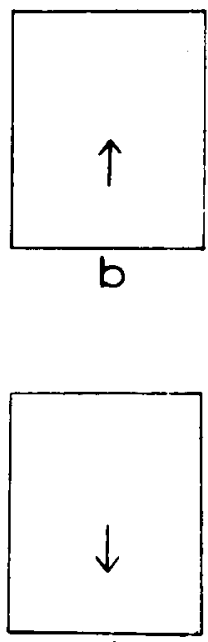

O
Figure 1. Four configurations used in this study: (a) an UP arrow in Position 1; (b) an UP arrow in Position 2; (c) a DOWN arrow in Position 1; and (d) a DOWN arrow in Position 2. 
"congruity" with its position (high vs. low). When, instead, the subjects had to decide whether the arrow was high or low in the rectangle, they yielded the analogous "congruity effect." These findings suggest that the height and vertical direction of an arrow are perceptually integral. and that the integrality is symmetrical.

If this height-direction integrality is symmetrical, however, it fails to fit Garner's hypothesis that one dimension will be integral with another "if in order for one dimension to exist the other must be specified." Every tangible object has a height, but not a direction. Height and direction should therefore yield asymmetrical integrality. since they are logically analogous to pitch and syllabicity in the Wood and Day study. Whereas irrelevant variation in height should interfere with judgments of direction, irrelevant variation in direction should not interfere with judgments in height. Our first empirical question was whether height and direction actually are symmetrical, as our earlier data suggest.

The integrality of height and direction, however, is unlike other types of integrality in a more important respect. In the Garner and Felfoldy study, subjects sometimes sorted color chips whose lightness and saturation were perfectly correlated. Compared to sorting without any variation on the irrelevant attribute, sorting here was facilitated. Apparently, when subjects could use information from both dimensions at once. they could discriminate between the color chips more quickly. Actually, there were two "correlated dimension" conditions, one with the lighter chips being the less saturated and a second with the lighter chips being the more saturated. It made no difference how the two dimensions were correlated, for there was facilitation in either case.

With height and vertical direction, however, the direction of the correlation appears to be critical. In our earlier study, subjects were faster in judging contigurations $a$ and $d$, those with congruity between position and direction, than in judging configurations $b$ and $c$, those without congruity. In a card sorting task like Garner and Felfoldy's, then, subjects should be quicker in sorting configurations $a$ and $d$ than in sorting contigurations $b$ and $c$, the two "correlated dimension" conditions. They should do better when height and direction are correlated "positively," high with UP and low with DOWN; than when they are correlated "negatively," high with DOWN and low with UP. The negatively correlated condition may even be harder than the conditions without any variation on the irrelevant dimension. If so, here is one instance where redundancy not only doesn't help, but actually hinders, and this is unlike other types of integrality. Our second and third empirical questions, then, were whether or not there is a disparity between the positively and negatively correlated conditions, as we expect, and if so, whether the negatively correlated condition actually hinders.
Given our previous findings, it is logically possible for position to facilitate or interfere with judgments of direction even when it does not vary. Consider subjects sorting configurations $a$ and $c$ into two piles. They should be fast in sorting $a$, in which height is in congruity with direction, but slow in sorting $c$, in which height is not in congruity with direction. On the other hand, since there is only one position for all arrows being sorted, subjects may be able to ignore position altogether and not be affected by it. Our fourth empirical question, then, was whethef or not there would be such a congruity effect when there was no variation on the irrelevant dimension.

Because of this last question, we could not use the card-sorting technique developed by Felfoldy and Garner (1971). In that technique, the investigator measures only how long subjects take to sort a deck of cards. He does not measure separately how long they take to sort one arrow into one pile and another arrow into another pile. We therefore had subjects "sort" configurations in blocks of 24 trials, but measured the latency for each decision separately.

\section{METHOD}

On each of 240 trials, subjects were shown an arrow within a rectangle and were asked to decide as quickly as possible either whether the arrow was pointing up or down ("direction judgments") or whether it was high or low in the rectangle ("position judgments"). The pictorial configuration shown on each trial was one of tour possible: (a) an UP arrow at Position 1 (just above the middle); (b) an UP arrow at Position 2 (just below the middle); (c) a DOWN arrow at Position 1; and (d) a DOWN arrow at Position 2. These four configurations are illustrated in Figure 1.

The total session consisted of 10 different tasks of 24 trials each. 5 tasks requiring direction jud gments and 5 tasks requiring position judgments. The 5 direction tasks differed in the subset of configurations they used: (1) a and c, UP and DOWN arrows both at Position 1: (2) b and d. UP and DOWN arrows both at Position $2 ;(3)$ a and $d$, UP arrows at Position 1, and DOWN arrows at Position 2; (4) b and $c$, UP arrows at Position 2. and DOWN arrows at Position 1 ; and (5) a, b, c, and d, both arrows at both positions. Thus 1 and 2 are both single dimensions tasks, 3 is a positively correlated dimensions task. 4 is a negatively correlated dimensions task, and 5 is an orthogonal dimensions task. The 5 position tasks were analogous and consisted of the following subsets: (1) a and b, UP arrows at Positions 1 and $2 ;(2) c$ and $d$. DOWN arrows at Positions 1 and 2; (3) UP arrows at Position 1 and DOWN arrows at Position $2 ;(4)$ b and $c$, DOWN arrows at Position 1 and UP arrows at Position 2; and (5) a, b, c, and d, both arrows at both positions. For each task there was a deck of 16 displays consisting of equal numbers of the two or four configurations in that task. Before each task, the deck was shuffled, and the subject was presented the first eight displays as practice trials. These eight displays were then placed at the end of the deck. and without a break the subject was given the whole deck for 16 test trials. The practice trials went unanalyzed.

The 10 tasks were presented to subjects in a counterbalanced order. For every 10 subjects, each task appeared in each serial position just once. This was achieved by use of a $10 \times 10$ Latin square (combining the 10 tasks, the 10 serial positions, and 10 subjects) that also constrained each task to follow each other task in only one of the 10 orders of presentation. At the start of the session there were two additional practice tasks to get the subjects used to the apparatus and procedure. One practice task required position judgments of unadorned lines (arrows without their heads) at 
Positions 1 and 2, and the other required direction judgments of UP and DOWN arrows in the very middle of the rectangle (half way between Positions 1 and 2). The two practice tasks went unanalyzed as well.

The subject started each trial by pressing a "ready" button. The configuration appeared before him $1 / 2 \mathrm{sec}$ later and remained on until he indicated his answer by pressing one of two response buttons labeled "up" and "down," or "high" and "low," depending on the instruction. The response latency was measured from the appearance of the display to the press of a button in milliseconds. The subjects pressed the "high"/"up" button with one thumb, and the "low"/"down" button with the other, and the "ready" button (situated between the other two buttons in a hand-held response panel) with either thumb. For half the subjects, "high"/ "up" was on the left, and "low"/"down" on the right; for the other half, the assignment was reversed. We did not include the response assignments "high"/"down" and "low"/"up" because they were potentially so confusing. We discuss the possible consequences of this confounding later.

The four configurations were drawn in black india ink on white. The enclosing rectangle was $4 \mathrm{~cm}$ wide and $5 \mathrm{~cm}$ high, and the arrow was $1 \mathrm{~cm}$ long. There were two complete sets of configurations differing only in the discriminability of Positions 1 and 2 . In Set $A$, the arrows at Positions 1 and 2 had their centers $.75 \mathrm{~cm}$ above and below the midline of the rectangle. In Set B, they were $1 \mathrm{~cm}$ above and below the midline. We included these two sets to look at certain effects of discriminability; as it turned out, the two sets yielded virtually identical results, so the two sets will be of no interest. All arrows were in the horizontal center of the rectangle. The configurations were viewed at $51 \mathrm{~cm}$ in the center of a $13 \times 7 \mathrm{~cm}$ window of a modified Iconix tachistoscope. The rectangle therefore subtended $5.6^{\circ}$ of visual angle. Between trials, the subjects looked at a $13 \times 15 \mathrm{~cm}$ blank adaptation field. The test field coincided with the lower $13 \times 7 \mathrm{~cm}$ portion of that field.

The $\mathbf{4 0}$ subjects we used were high school and college age men and women earning 52 or fulfilling a course requirement for introductory psychology during the summer session at Stanford University. The 20 subjects receiving Set A filled two complete replications of the $10 \times 10$ Latin square design, one replication with the "high"/"up" button on the left, and the other with the "high"/"up" button on the right. The 20 subjects receiving Set B fitted the same design. One additional subject was eliminated for making more than $10 \%$ errors. Before each block of trials, the subjects were told whether the task required position judgments or direction judgments. They were encouraged to respond as quickly as possible without making errors.

\section{RESULTS}

We analyzed the latencies in two ways. One was to view the experiment as a card-sorting experiment à la Garner and Felfoldy with only one mean latency per task. The other was to view the individual displays as critical and to examine the differences between displays within each task as well. Obviously, the

Table 1

Mean Latencies (in Milliseconds) for the Five Direction Tasks

\begin{tabular}{lc}
\hline \multicolumn{1}{c}{ Task Name } & Mean \\
\hline Single dimension (Position 1) & 471 \\
Single dimension (Position 2) & 453 \\
Positively correlated dimensions & 436 \\
Negatively correlated dimensions & 520 \\
Orthogonal dimensions & 511 \\
Mean & 478 \\
\hline
\end{tabular}

Table 2

Mean Latencies (in Milliseconds) for the Five Position Tasks

\begin{tabular}{lc}
\hline \multicolumn{1}{c}{ Task Name } & Mean \\
\hline Single dimension (UP arrows) & 441 \\
Single dimension (DOWN arrows) & 438 \\
Positively correlated dimensions & 428 \\
Negatively correlated dimensions & 454 \\
Orthogonal dimensions & 465 \\
Mean & 445 \\
\hline
\end{tabular}

analyses by task and by display are not independent, but they do raise different issues. For both analyses, we averaged the latencies of each subject over the correct responses to each display within each task and used the resulting 960 means as our basic units of analysis.

\section{Analysis by Task}

The mean latencies for the five direction tasks are shown in Table 1, and those for the five position tasks in Table 2. In an analysis of variance incorporating all these latencies, there were only three reliable effects. The five tasks differed from each other significantly, $F(4,152)=23.66, p<.001$. The position judgments were $33 \mathrm{msec}$ faster, on the average, than the direction judgments, $F(1.38)=31.61, p<.001$. And there was a reliable interaction between tasks and types of judgments, $F(4,152)=4.48, p<.005$. Somewhat surprisingly, there were no reliable main effects or interactions that involved Sets $A$ and B, and so we can deal solely with the 10 means in Tables 1 and 2. Furthermore, among those 10 means, the five tasks accounted for $57.0 \%$ of the variance, type of judgment (position vs. direction) $30.4 \%$, and their interaction only $12.6 \%$. Hence we are justified in concentrating on the differences among the five tasks.

The two single dimension tasks, viewed as controls, were not reliably different in either the direction or the position judgments, $F(1,152)=3.04$ and $F<1$, respectively. Overall, they averaged $451 \mathrm{msec}$. Compared to this mean, the positively correlated tasks $(432 \mathrm{msec})$ were significantly faster, whereas the negatively correlated tasks $(487 \mathrm{msec})$ and the orthogonal dimensions tasks $(488 \mathrm{msec})$ were each significantly slower. The positively correlated tasks $(432 \mathrm{msec})$ were therefore significantly faster than the negatively correlated tasks $(487 \mathrm{msec})$. These four comparisons yielded $F(1,152)=9.03,32.42,34.25$, and 56.75, respectively, with the first $p<.005$ and the other three $p<.001$. For the direction judgments alone, these four comparisons were all highly significant, $F(1,152)=8.46,42.08,20.02$, and 66.19 . respectively, all at least $\mathrm{p}<.005$. For the position judgments alone, however, the same four comparisons were smaller, $F(1.152)=1.51,2.81,8.46$, and 6.34 ; only the last two comparisons are significant, at $\mathrm{p}<.005$ and $\mathrm{p}<.025$, respectively. Thus it is the 
Table 3

Mean Latencies (in Milliseconds) of Each Display in the Direction Judgments*

\begin{tabular}{|c|c|c|c|c|c|c|c|c|c|}
\hline \multirow{3}{*}{\begin{tabular}{l}
\multicolumn{1}{c}{ Task } \\
Single dimension \\
Correlated dimensions \\
Orthogonal dimensions
\end{tabular}} & \multicolumn{4}{|c|}{ Position 1} & \multicolumn{4}{|c|}{ Position 2} & \multirow{3}{*}{$\begin{array}{r}\begin{array}{c}\text { Congruity } \\
\text { Effect }\end{array} \\
+0 \\
+84 \\
+42\end{array}$} \\
\hline & \multicolumn{2}{|c|}{ UP } & \multicolumn{2}{|c|}{ DOWN } & \multicolumn{2}{|c|}{ UP } & \multicolumn{2}{|c|}{ DOWN } & \\
\hline & $\begin{array}{l}464 \\
429 \\
485\end{array}$ & $\begin{array}{l}(1.6) \\
(.3) \\
(2.5)\end{array}$ & $\begin{array}{l}477 \\
524 \\
525\end{array}$ & $\begin{array}{l}(1.6) \\
(2.8) \\
(4.4)\end{array}$ & $\begin{array}{l}447 \\
516 \\
542\end{array}$ & $\begin{array}{l}(1.6) \\
(4.4) \\
(8.8)\end{array}$ & $\begin{array}{l}458 \\
443 \\
497\end{array}$ & $\begin{array}{l}(1.2) \\
(.3) \\
(0)\end{array}$ & \\
\hline Mean & 460 & & 509 & & 502 & & 466 & & +42 \\
\hline
\end{tabular}

*Percentage errors in parentheses

consistently larger variation in the direction tasks that accounts for the significant interaction between tasks and judgments noted earlier.

\section{Analysis by Display}

Each of the four displays (the UP arrow at Position 1. for example) appeared in three direction tasks-a single dimension task, a correlated dimensions task, and the orthogonal dimensions task. That is, it appeared in the context of another arrow at the same position, in the context of another arrow at the opposite position, and in the context of all three remaining displays. Each display also appeared in three position tasks, one with single dimensions, one with correlated dimensions, and one with orthogonal dimensions. The mean latencies for each display classitied this way are listed for the direction judgments in Table 3 and for the position judgments in Table 4.

The main point of this analysis was to examine the "congruity effect." In our previous work (Clark \& Brownell, 1975). subjects were faster in direction judgments on UP arrows that were high in the rectangle than on those that were low; and they were faster on DOWN arrows that were low in the rectangle than on those that were high. Arithmetically, the congruity effect in direction judgments is the mean of these two differences in latencies. The congruity effect in position judgments is analogous. In this analysis, unlike the earlier study, we can compare the congruity effects of displays in the three distinct contexts-in single dimension tasks, in correlated dimensions tasks, and in orthogonal dimensions tasks. The sizes of these congruity effects are shown separately for direction and position judgments in Tables 3 and 4 . Note that in the correlated tasks, the congruity effect is exactly equivalent to the difference between the positively correlated task and the negatively correlated task in the earlier analysis by tasks; the $\$ 4-\mathrm{msec}$ congruity effect in Table 3 matches the $84-\mathrm{msec}$ difference between the two tasks in Table 1. In effect, then, we are separating out the positively and negatively correlated displays in the single dimensional tasks and in the orthogonal tasks the same way we did in constructing the two correlated dimension tasks.

Taken together, the direction and position judgments yielded a highly reliable congruity effect. averaging $34 \mathrm{msec}, \mathrm{F}(1,38)=81.87, \mathrm{p}<.001$. The congruity effect was slightly larger for direction judgments $(42 \mathrm{msec})$ than for position judgments $(25 \mathrm{msec}), \mathrm{F}(1,38)=5.29, \mathrm{p}<.05$, but both were highly reliable by themselves, $F(1,48)=63.82$ and 22.61, respectively, $\mathrm{p}<.001$. What is important, however, is that the congruity effect varied considerably with the classification of the displays. With direction and position judgments averaged together. the single dimensions tasks yielded the snallest congruity effect $(7 \mathrm{msec})$, the orthogonal dimensions tasks the next largest $(38 \mathrm{msec})$, and the correlated dimensions tasks the largest $(55 \mathrm{msec})$, $F(2,76)=14.04, p<.001$. But this variation was due almost entirely to the direction judgments, as shown in the reliable interaction between judgments and display classification, $F(2.76)=6.70, p<.005$. Taken separately, the direction judgments yielded a highly significant variation in the congruity effect, $F(2,76)=20.74, p<.001$, whereas the position judgments did not, $F(2,76)=1.31$, n.s. So, for direction judgments, there is no congruity effect when position doesn't vary over trials, but there is a substantial one when it does. For position judgments. on the other hand, there is approximately the same congruity effect no matter whether the arrow's direction varies over trials or not.

As in the analysis by tasks, Sets A and B yielded

Table 4

Mean Latencies (in Milliseconds) of Each Display in the Position Judgments*

\begin{tabular}{|c|c|c|c|c|c|c|c|c|c|}
\hline & \multicolumn{4}{|c|}{ UP } & \multicolumn{4}{|c|}{ DOWN } & \multirow{2}{*}{$\begin{array}{c}\text { Congruity } \\
\text { Effect }\end{array}$} \\
\hline & \multicolumn{2}{|c|}{ Position 1} & \multicolumn{2}{|c|}{ Position 2} & \multicolumn{2}{|c|}{ Position 1} & \multicolumn{2}{|c|}{ Position 2} & \\
\hline $\begin{array}{l}\text { Single dimensions } \\
\text { Correlated dimensions } \\
\text { Orthogonal dimensions }\end{array}$ & $\begin{array}{l}430 \\
427 \\
442\end{array}$ & $\begin{array}{l}(.6) \\
(1.9) \\
(0)\end{array}$ & $\begin{array}{l}451 \\
461 \\
485\end{array}$ & $\begin{array}{l}(1.2) \\
(1.6) \\
(3.1)\end{array}$ & $\begin{array}{l}442 \\
447 \\
481\end{array}$ & $\begin{array}{l}(1.2) \\
(1.2) \\
(1.9)\end{array}$ & $\begin{array}{l}435 \\
430 \\
454\end{array}$ & $\begin{array}{l}(1.2) \\
(1.9) \\
(1.2)\end{array}$ & $\begin{array}{l}+14 \\
+26 \\
+35\end{array}$ \\
\hline Mean & 433 & & 466 & & 457 & & 440 & & +25 \\
\hline
\end{tabular}

*Percentage errors in parentheses 
almost identical patterns, and so they have not been listed separately in Tables 3 and 4. Actually, three small interactions with Sets $A$ and $B$ reached statistical significance, but they were only at the .05 level and none was larger than $9 \mathrm{msec}$. All were higher order interactions with no ready interpretation and did not in any way alter the trends in the congruity effects we just examined.

The error rate overall was $1.8 \%$, ranging from $0 \%$ to $6.9 \%$ for individual subjects. The error rates for each display are shown in Tables 3 and 4 , but are so low and unreliable that we did not analyze them any further. Overall, the error rates by display correlated .52 with mean latencies, indicating at least that there was no severe speed-accuracy tradeoff that might go against our conclusions.

\section{DISCUSSION}

We have demonstrated that the position and direction of an arrow are perceptually integral dimensions of a rather novel variety. Like other integral dimensions, position and direction were judged more slowly when there was orthogonal variation along the other, irrelevant dimension. But unlike most other integral dimensions, position and direction were judged more quickly when there was positively correlated variation along the irrelevant dimension, UP with high and DOWN with low, but more slowly when there was negatively correlated variation, UP with low and DOWN with high. Here, then, is one case where providing the subjects with a redundant dimension helped or hindered.depending on whether the two dimensions were correlated positively or negatively.

The integrality of position and direction appears to be roughly symmetrical. For our configurations, the two positions were slightly more discriminable than the two directions UP and DOWN, for there was a 440- to 462-msec advantage favoring position in the single dimension tasks, $F(1,38)=5.68, p<.025$. Since position was more discriminable than direction, position should more easily "intrude" on direction judgments than vice versa (Clark \& Brownell, 1975; Morgan \& Alluisi, 1967), and it did. Orthogonal variation in position slowed down direction judgments by $49 \mathrm{msec}$, whereas orthogonal variation in direction slowed down position judgments by only $25 \mathrm{msec}, \mathrm{F}(1,152)=9.23, \mathrm{p}<.005$. The positively correlated task exceeded the negatively correlated task by $84 \mathrm{msec}$ in direction judgments, but by only $26 \mathrm{msec}$ in position judgments, $F(1,152)$ $=53.88, \mathrm{p}<.001$. And the overall congruity effect was larger for direction judgments (with position intruding) than for position judgments (with direction intruding), 42 to $25 \mathrm{msec}$. In this study, then, position was more discriminable and therefore more intrusive. In Experiments 3 and 4 of Clark and Brownell (1975), it was direction that was the more discriminable and also the more intrusive.

These findings are difficult to square with Garner's proposal, which leads to the prediction that position should intrude on direction judgments, but not vice versa. We found interference on both sides, and with no hint even that position is more intrusive than direction. So, for these two dimensions at least. discriminability seems to be more important than intrinsicality in determining how much one dimension will intrude on the other.

Unexpectedly, however, position and direction judgments diverged in one qualitative aspect. In direction judgments, the congruity effect was nil $(0 \mathrm{msec})$ in the single dimension tasks, moderate $(42 \mathrm{msec})$ in the orthogonal dimensions tasks, and very large $(84 \mathrm{msec})$ in the correlated dimensions tasks. For the position judgments, in contrast, the congruity effect was virtually the same in all three types of tasks, 14, 35, and 26 msec. Why? The explanation may lie in the nature of position and direction as dimensions.

In our configurations, position is relative, and direction is absolute. An arrow is high or low only relative to some outside standard. When height changes from trial to trial. the observer should take the middle of the rectangle as that standard, "seeing" the arrow as either high or low. But when height doesn't change, he can take the level of the arrow itself as the standard, "seeing" the arrow as neither high nor low. If this is so, height should intrude on direction judgments in the first case, but not in the second. This is just what we found. Likewise, an arrow points up or down relative to some standard direction. But no matter whether direction does or doesn't change from trial to trial, the observer has only the directionality inherent in the display to take as the standard, and so he will always "see" an UP arrow as pointing up and a DOWN arrow as pointing down. If this is so, direction should intrude on position judgments about equally in the two cases. This, too, is just what we found. This explanation, however, should be taken with some caution, for it is post hoc and requires further testing.

In this study, "up" was always assigned to the same hand as "high," and "down" to the same hand as "low." Might this, along with the rapid succession of the 10 tasks, have fostered a correlation between high-low and up-down and thereby produced our findings artificially? We think not. In one of our earlier tasks (Experiment 1. Clark \& Brownell, 1975), subjects judged arrows as pointing "up" or pointing "down" for configurations like those in Figure 1. Without any mention of "high" or "low" in the experiment, there was a substantial congruity effect. In a second task (Experiment 2), subjects read a one-word instruction $u p$ or down and then judged each conniguration as "true" or "false" depending on 
whether the instruction was true or false of the arrow's direction. Without any mention of "high" and "low" and without even the overt responses "up" and "down," there was again a substantial congruity effect; furthermore, it had the same size and form as the previous one. The response system does not seem to be the source of the congruity effect (see Clark \& Brownell, 1975).

Evidence internal to the present study suggests that there was little, if any, contamination from task to task. A pure contamination explanation would predict the congruity effect to be just as large in the single dimension tasks as in the correlated dimension tasks (Tables 3 and 4). Yet, in the direction judgments, the congruity effects were 0 and $84 \mathrm{msec}$, respectively, a very large difference for these data. This explanation would have special trouble with the $0 \mathrm{msec}$ effect here, because any contamination at all from task to task would make this greater than 0 . The 0 - vs. 84 -msec difference. indeed, underlies the main result of the study: positive correlations facilitate, and negative correlations interfere. In the position judgments, the two effects were 14 and $26 \mathrm{msec}$, respectively, more in line with the contamination explanation. But why should contamination work from up-down to high-low and not vice versa? Nor can contamination explain the other differences between the position and the direction judgments. So, even if there was some contamination from tasks to task, it does not compromise the main findings.

At least two other studies have noted that direction of correlation makes a difference, but both studies turned up results exactly opposite to our own. Pomerantz and Garner (1973) had subjects sort the four figures $((, 0),($, and $))$ into piles. They defined the two dimensions in these figures to be the first and second parentheses and compared, among other things, sorting of the two positively correlated figures $(($ and $))$ with sorting of the two negatively correlated figures () and )(. Subjects found the latter much easier to sort. and according to Pomerantz and Garner (1973) that was because the figures had emergent properties that made sorting easier. Similarly, Lappin, Bell, Harm, and Kottas (1975) had people judge the spatial and temporal intervals between two dots. Subjects were much faster when the space and time intervals were negatively correlated, making the dots appear to move at very different velocities, than when the two intervals were positively correlated, making the dots appear to move always at the same velocity. As Lappin et al. argued, their subjects used the emergent property, apparent dot velocity, to discriminate between configurations more easily. In any event, both studies-both types of integrality-contrast with the present type.

Height and direction get their integrality from the observer's "interpretations" of perceptual information. To define a "positive" or "negative" correlation between two dimensions, we must be able to define a directionality, an increasing scale, along both dimensions. For example, we cannot define directionality for hue (red. vs. blue) or shape (rectangle vs. circle), since neither dimension lies on a directed scale. Indeed, directionality is a rather abstract property. Nothing intrinsic to the shape of an UP arrow says it is "increasing in value" in an upward direction; rather, it is a symbol we take as standing for something moving or pointing upward. And nothing intrinsic to an arrow's position says it is positive in height; for this we must impute up-down directionality to the surrounding rectangle and compare the arrow's position to a point along that scale. Only when the arrow's shape is interpreted as vertical direction and its position as height can the two perceptual codes facilitate and interfere with each other from elements they have in common or in conflict (see Clark \& Brownell, 1975).

Where else should positive correlations facilitate and negative correlations interfere? It may be critical that two dimensions have a central component of interpretation in common, just as height and vertical direction have "verticality" in common. Height and horizontal direction, for example, should not show any strong congruity effects. In informal experiments, we have found congruity effects for size and height, with the component "extent" in common, but not for length and vertical direction, with no obvious component in common. But it may not be critical that the two dimensions belong to the same stimulus, as the word integrality presupposes. Other investigators (e.g., Bernstein \& Edelstein, 1971; Simon \& Craft, 1970) have found facilitation and interference even when the two dimensions were in separate stimuli and in different modalities-for example, visual height and auditory frequency (which is described as height). In this way, these findings are also akin to the well-known Stroop effect (see Clark \& Brownell, 1975).

Ultimately, these phenomena bear on several important questions. What is the nature of the perceptual code? When are two dimensions interpreted with coding components in common? How are the various types of integrality related to the varieties of the Stroop phenomenon? When are two dimensions combined into an emergent dimension? We need to answer these questions before we can make sense of perceptual integrality in all its guises.

\section{REFERENCES}

Bernstein. I. H., \& Edelstein, B. A. Effects of some variations in auditory input upon visual choice reaction time. Journal of Experimental Psychology, 1971, 87, 241-247.

Clark. H. H.. \& Brownell. H. H. Judging up and down. Journal of Experimental Psichology: Human Perception and Pertormance. 1975, 1. 339.352. 
Felfoldy. G. L.. \& Garner, W. R. The effects of speeded classification of implicit and explicit instructions regarding redundant dimensions. Perception \& Psychophysics. 1971, 9. 289-292.

GARNER, W. R. Information integration and form of encoding. In A. W. Melton \& E. Martin (Eds.), Coding processes in human memory. Washington. D.C: Winston, 1972.

GARNER, W. R. The processing of information and structure. Potomac, Md: Lawrence Erlbaum Associates, 1974.

GARNer. W. R., \& Felfoldy, G. L. Integrality of stimulus dimensions in various types of information processing. Cognitive Psychology, 1970, 1, 225-241.

Lappin, J. W., Bell, H. H., HaRm, O. J., \& Kottas, B. On the relation between time and space in the visual discrimination of velocity. Journal of Experimental Psychology: Human Perception and Performance. 1975. 1. 383-394.
Morgan, B. B., \& Alluisi, E. A. Effects of discriminability and irrelevant information on absolute judgments. Perception \& Psychophysics, 1967, 2, 54-58.

Pomerantz, J. R., \& Garner, W. R. Stimulus configuration in selective attention tasks. Perception \& Psychophysics, 1973. 14, 565-569.

Pomerantz, J. R., \& SAger, L. C. Asymmetric integrality with dimensions of visual pattern. Perception \& Psychophysics, $1975,18,460-466$.

Simon, J. R., \& CRAFT. J. L. Effects of an irrelevant auditory stimulus on visual choice reaction time. Joumal of Experimental Psychology, 1970, 86, 272-274.

(Received for publication August 18, 1975; revision received December 3, 1975.) 